TM-1420

1620.000

1670.000

5520.000

(SSC-N-243)

\title{
NMR MEASUREMENTS IN SSC DIPOLE D00001
}

\author{
M. Kuchnir, E. E. Schmidt, R. W. Hanft, and J. B. Strait \\ Fermi National Accelerator Laboratory \\ Batavia, Illinois 60510
}

September 12, 1986 


\section{Fermilab}

TM-1420

1620.000

1670.000

5520.000

September 12, 1986

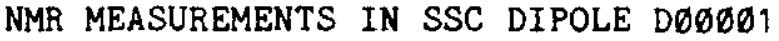

M. Kuchnir, Edward E. Schmidt, R.W. Hanft and J.B. Strait

Fermi National Accelerator Laboratory

Batavia, Illinois 60510

ABSTRACT

The first $16.5 \mathrm{~m}$ long SSC dipole magnet (Døøøø1) had its fieid intensity measured as a function of position with a custom made NMR magnetometer.

A short description of the probe is presented. The data obtained (most of it near $2 \mathrm{~T}$ spaced apart by one inch) shows an average transfer function of $1.02830 \mathrm{~T} / \mathrm{KA}$ with position dependent values deviating from the average by up to $.00130 \mathrm{~T} / \mathrm{KA}$ revealing construction inhomogeneities that were measured with a sensitivity of $25 \mathrm{ppm}$. 


\section{INTRODUCTION}

In order to allow measurements of magnetic pield harmonic content, the test set up of the first full size SSC dipole to be measured in superconducting state, includes a warm bore tube 838" long with 1.136" I.D. speclally designed for the harmonlc content measuring probe ("mole")." We bullt a NMR probe that would fit in this warm bore tube and used the same fishing gear + measuring tape arrangement that was used for preliminary longltudinal positioning of the mole.

The "committee approved" schedule for the sequence of measurements to be carried out in this first magnet called for transfer function measurements using NMR at a current of $2 \mathrm{KA}$ to be carried out after harmonic measurements were complete and before the magnet was further tested. This is the report on these measurements that were carrled out on Aug. 22, Aug. 27 and Sept. 4, 1986.

\section{HISTORICAL PRESENTATION OF THE DATA}

The first time avallable for testing the measuring equipment was on Aug. 22. It was used for checking the mechanical fit of the probe in the bore tube, the operation of its aligning magnet, the compatibility with the mole's fishing gear, and the detection of an NMR signal for the first time with this probe. Lack of operational data acquisition software prevented more than a few points to be measured, also a considerable increase in the noise level with depth into the warm bore was observed, which was attributed to the probe casing getting electrically shorted to the warm bore tube, due to easy damage of its $.0015^{\prime \prime}$ thick Kapton insulation layer.

With the external diameter of its copper casing turned down by $.015^{\prime \prime}$ and isolated by .004" thick Kapton tape along with a spiral of $.010^{\prime \prime}$ thick Tefion tape, shorts to bore tube were prevented and the first version of data acquisition software allowed us to prove on Aug. 27 that the system was "operational" and useful by taking data at $3^{\prime \prime}$ intervals from a depth of 360" into the bore tube. See curve $A$ in Figure 1. Upon closer examination at 1/2" measuring intervals at a region around 295" within the magnet revealed data point reproducibilities within $20 \mathrm{ppm}$ and structure in the field intensity as narrow as 2" wide. See Figure 2.

The next step was for us to make and use a longer cable ( $81 \mathrm{ft}$ ) capable of measuring the entire magnet in a single pass. Concern about getting the electronics to work properly with the extra capacitance of such a cable (much longer than the $35 \mathrm{ft}$ one used for measuring the Energy Saver magnets) led us to use first a $46.5 \mathrm{ft}$ cable with the idea of using it twice from opposite ends to cover the whole magnet.

With the longer cable, and a slightly modified program (position shown to operator in feet and inches and transfer function displayed for every data point) an essentially complete sweep of the magnet was carried on Sept. 4, 1986 in 1" increments. See curve B in Figure 1 and 
Figure 2 or the computer plot in Figure 3. A slight change (elimination of a gain 100 amplifier) in the current measuring clrcuit accounts for the shift of $250 \mathrm{ppm}$ between both curves indicating that the amplifier gain was actually 100.025 .

On this occasion with the probe 108" into the bore tube data was acquired on the transfer function stablilty in time (See Figure 4) and dependence on magnet current (See Figure 5).

\section{PROBE DESCRIPTION}

The electronics of the probe is essentially the same as one of the probes bullt at Fermilab for measuring the Energy Saver magnets. It is very much a copy of the one developed by $\mathrm{K}$. Borer and G. Fremont ${ }^{2}$. They differ from it in:

1. The use of gum rubber ${ }^{3}$ instead of doped water as the sensitive material.

2. The inclusion of a Hall probe in the sensor in order to extend its range and facilitate tuning into the narrow frequency band of the NMR signal, which enables the mapping of the end fields and the easy observation of probe alignment.

3. The inclusion of part of the electronics into the probe casing for mechanical protection and simplified electrical shielding.

The particular probe custom made for the SSC is encased in a cylindrical instead of prismatic metal box. This cylindrical box is shown in Figure 6 , it is rigidly attached to an internal coil which will align the sample perpendicular to the magnetic field to be measured, maximizing the signal/noise ratio. This aligning coil consists of 100 turns of $26 \mathrm{AWG}$ enamel insulated magnet wire wrapped around a $3 " \mathrm{x} 0.75^{\prime \prime}$ coil form. A maximum torque of 2.5 in-1b is generated by 1 A flowing through it when in a $2 \mathrm{~T}$ field. Its effect in the NMR reading was below the noise level. The sample volume is a cylinder $15 \mathrm{~mm}$ long by $5 \mathrm{~mm}$ diameter located as indicated in Figure 6.

Mechanically the extremities of the probe have \#8-32 screw studs for attaching to the fishing lines of the temporary mole positioning system. A $1 / 4^{n}$ compression fitting in one extremity is connected to the polyflow tubing that brings warm $\mathrm{N}_{2}$ gas to keep the probe temperature near room temperature when inside the bore tube. This gas flows through the inside of the probe and exits through a series of small holes in its cylindrical copper wall. The opposite extremity has two connectors besides the fishing line attachment with which the probe is pulled manually: a SMA for the coaxial cable carrying the radio frequency signal and a 10 pin LEMO carrying feedback, power and other utilities for the probe electronics; Hall probe and aligning coil.

A surveyors measuring tape, made out of cloth and graduated in $1 / 8$ " increments is taped to the cables with its zero corresponding to the 
center position of the sample. It is used to determine the sample position in relation to the south extremity of the mole warm bore tube in the test stand. This improvised position measuring system is estimated to yield the position of the probe within $\pm 1 / 8^{n}$ neglecting the systematic errors due to stretching tension and thermal contraction.

The program for data acquisition is also rather simple, although it is interfaced to the sophisticated data acquisition and control sof tware of the test. Two operators near the south end of the testing stand are needed for efficlent NMR data collection; one manually pulls the probe to its new position and notifies the other who then tunes in manualiy the NMR signal and presses a button commanding the software to read the magnetic field that is digitally indicated in the NMR electronic NIM control module. The software also reads the time and the current running through the magnet. The software then notifles acceptance of the data by a beep, and through a remote terminal screen near the operators displays the data just recorded and prompts them with the position for the next point. Figure 7 shows a sample of the 5 th file MDTF: :Døø001. NA05_NMR generated and named by this VAX 11/730 sof tware. At the start of a run the operator is prompted for the starting position and the incremental position step. The file is closed upon exiting the program and there is provision for redoing part of the run in case the operators find themselves out of step in the positioning of the probe.

\section{DATA INTERPRETATION}

The reproducibility observed in Figures 1,2 and 3 indicates that the very irregular pattern is real and characteristic of this magnet. The dip $32^{n}$ south of the center is about $1 \mathrm{ft}$ wide in average and $.06 \%$ below magnet average, a non-negligible amount. As can be seen in Figure 1 no correlation exists with the position of the supporting posts or welds.

The intervals between dips are probably related with the coil manufacturing technique. A $5 \mathrm{ft}$ interval is rather pronounced and a Fourier analysis of curve $B$ is bound to show others that could best be interpreted by the magnet manufacturers.

Examination of Figure 2 demonstrates the sensitivity and reproducibility of this technique. Since the sample being used is less than $5 \mathrm{~mm}$ long along the axis of the magnet, much higher discrimination can be obtained with a more elaborate probe positioning and ranging procedure.

The data points in Figure 5 have been numbered to indicate the sequence of the measurements. The apparent misalignment of points \#2 and \#3 acquire special meaning when it is revealed that a quench occurred after $\# 1$ was measured, that $\# 2$ was reached under an increasing-current condition and \#3 after current reversal with the others following under decreasing-current conditions. A behavior consistent with the existence of persistent current hysteresis. One would expect these points to be in a horizontal line indicating current 
independence. The observed slope of -0.0021 ( $T / K A$ )/KA is sign wise consistent with heating of the shunt used in measuring the current and expansion of the magnet coll under Lorenz forces against ylelding collars.

\section{ACKNOWLEDGEMENTS}

We thank J.E. Pachnik for the data acquisition software, D. Hartness and $K$. Dillow for help with the measurements.

\section{REFERENCES:}

1. P. Wanderer, "Production Techniques for Measuring SSC Accelerator Magnets," Proc. of the ICFA Workshop on Superconducting Magnets and Cryogenics, Brookhaven National Laboratory (1986)

2. K. Borer and G. Fremont, The Nuclear Magnetic Resonance Magnetometer, type 9298, Publication 77-99, CERN.

3. Gum rubber sample is cut from the wall of a tack-free latex tubing similar to catalog number R6402-09 of Cole-Palmer Instrument Company, 7425 N. Oak Park Ave., Chicago, Il 60648. 


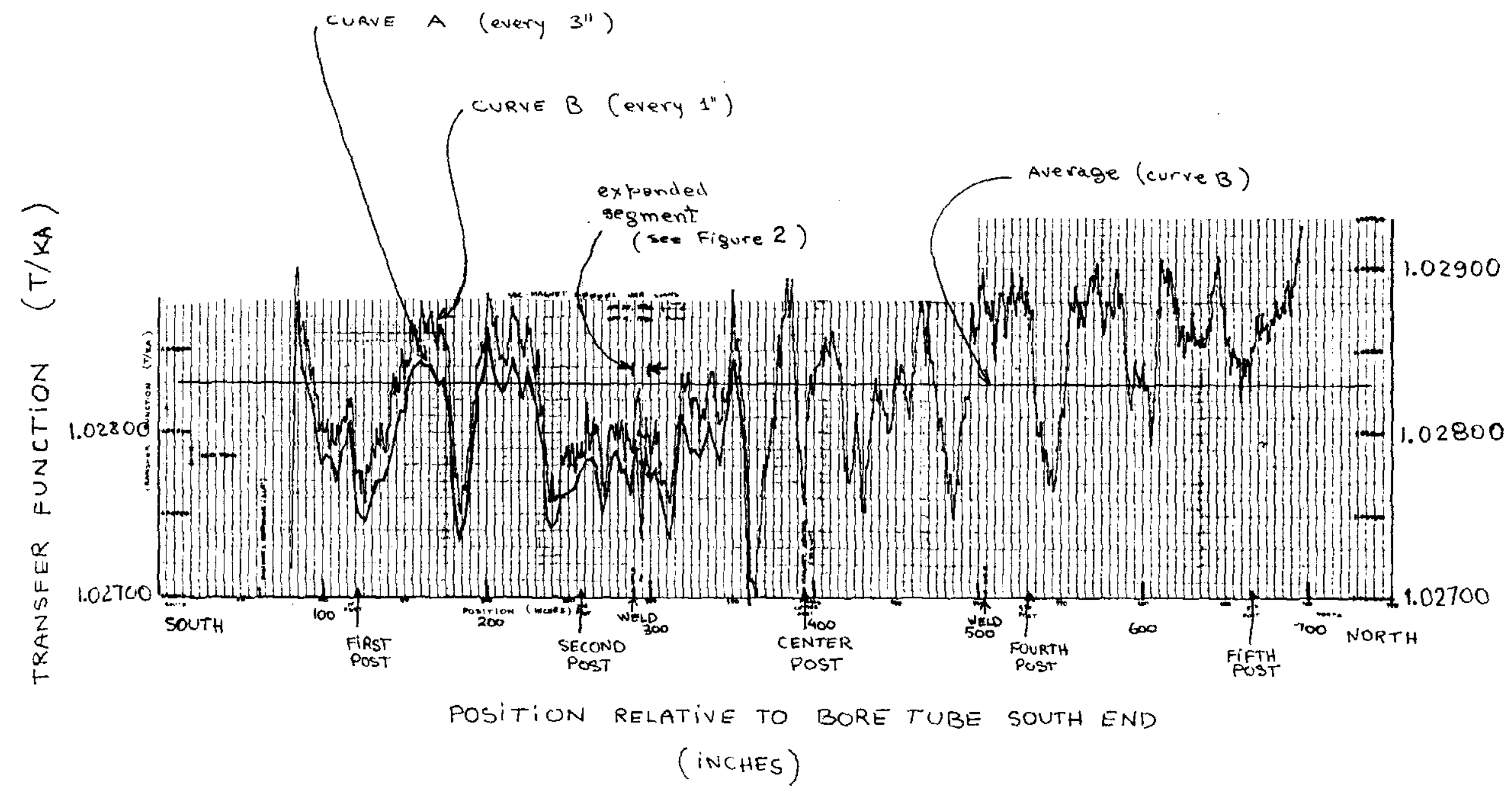

Figure 1 Transfer function dependence on position of SSC dipole D00001 near 2 Teslas. 


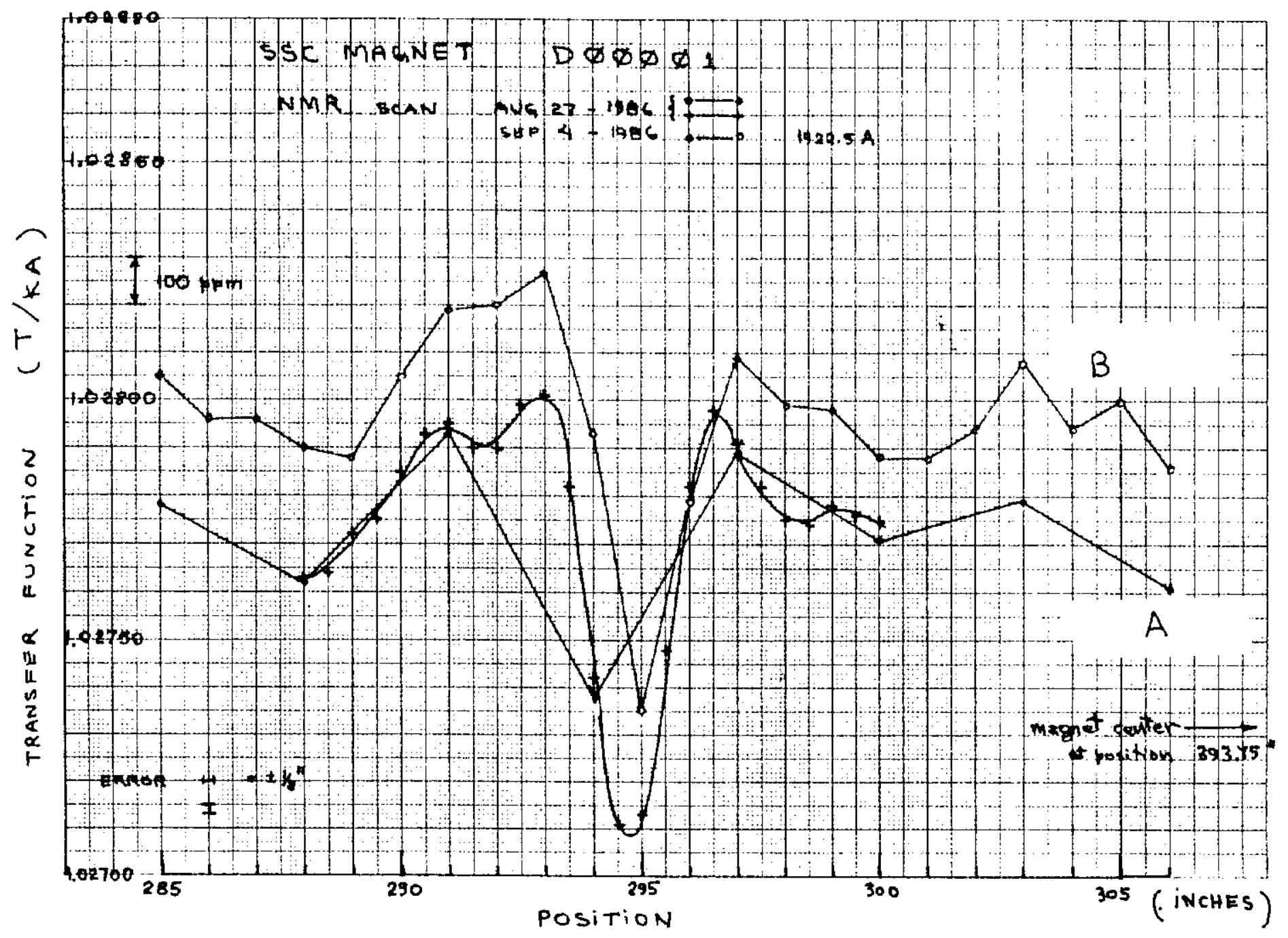

Figure 2 Expanded detail of transfer function position dependence. 


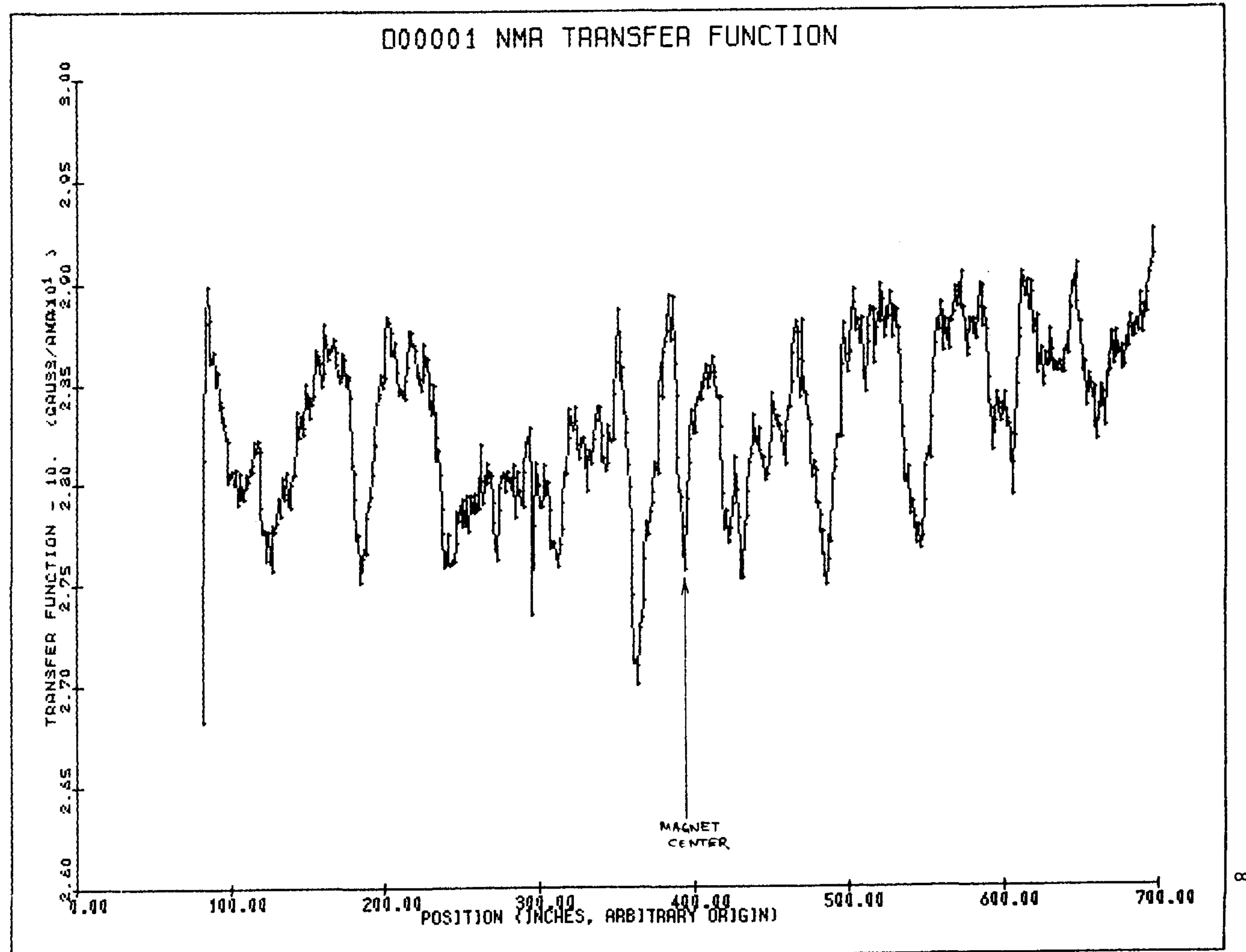

Figure 3 Transfer function of $0 \emptyset \emptyset \emptyset \emptyset 1$ (curve B onlv). 


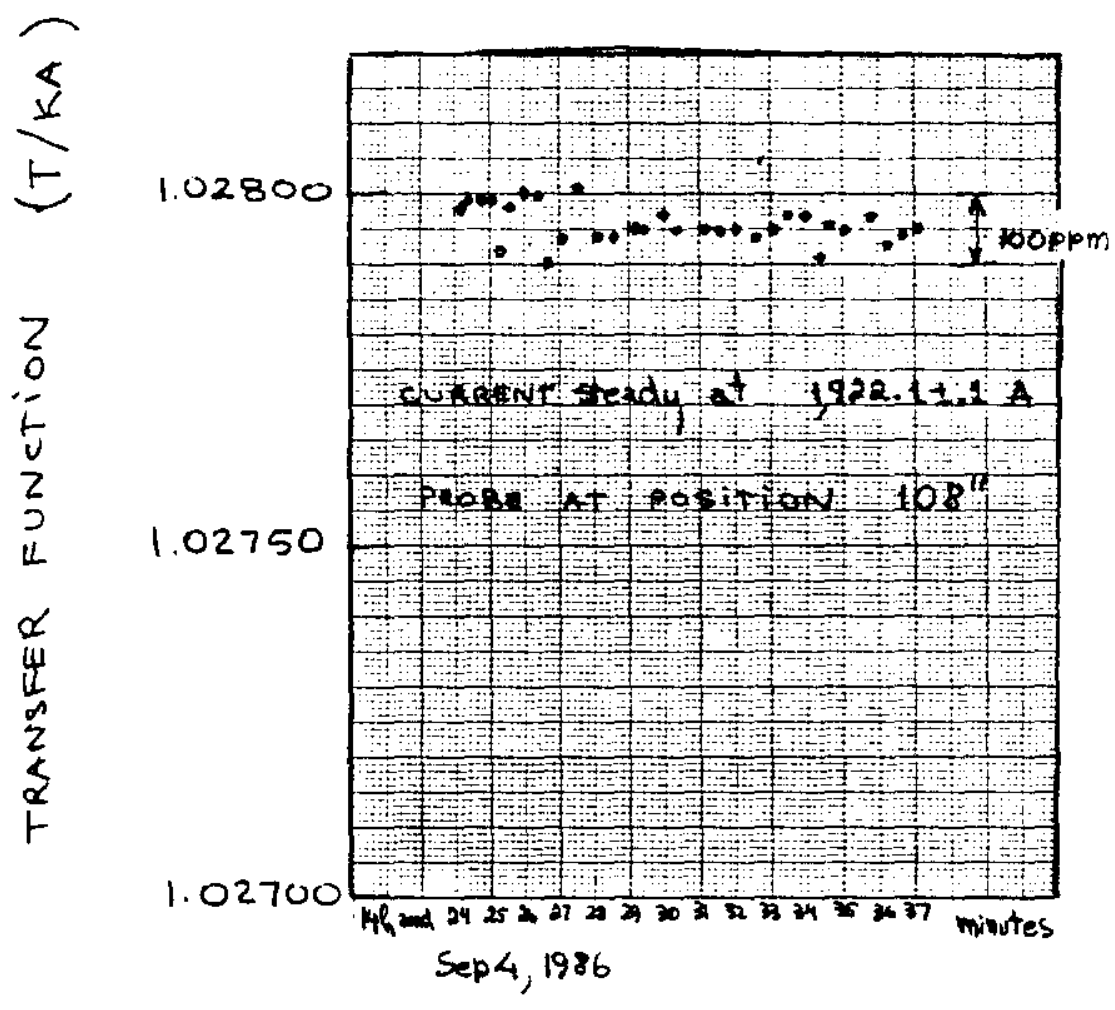

TIME

Figure 4 Time dependence of transfer function. 


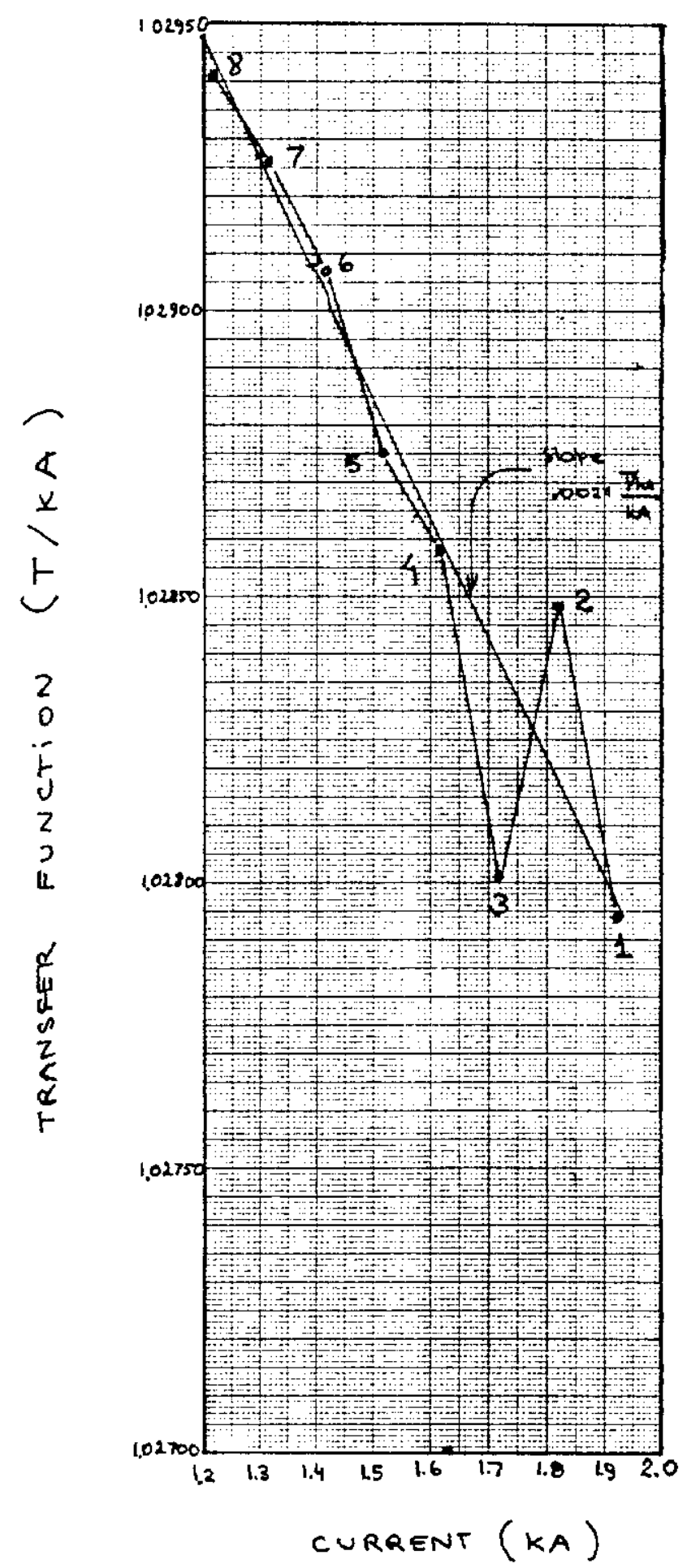

Figure 5 Current dependence of transfer function. 


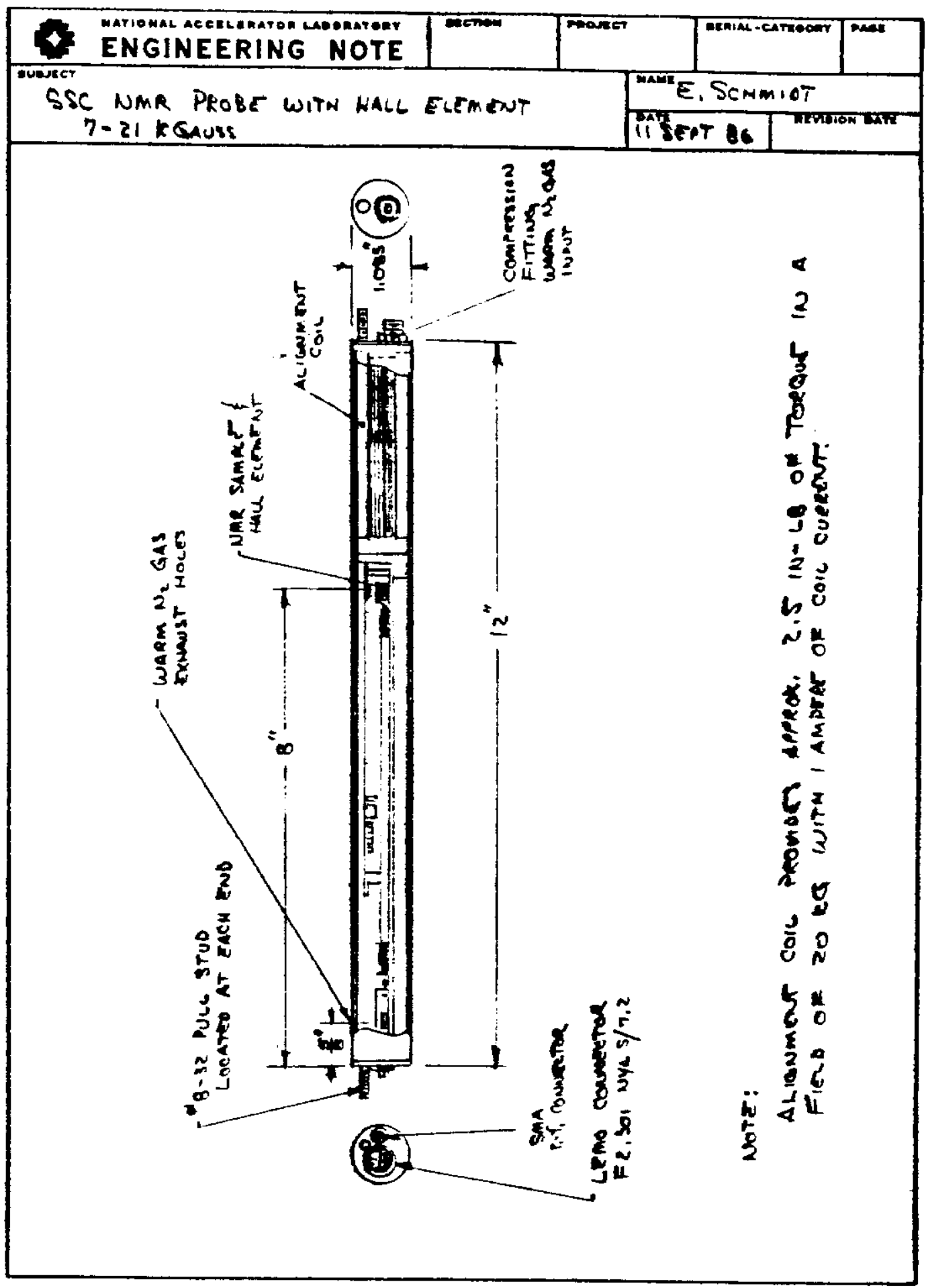

Figure 6 NMR magnetometer probe for SSC dipoles. 
D00001

$\begin{array}{rc}\text { POINT } & \text { B } \\ 1.0 & 19788.4 \\ \text { 2. } & 19785.8 \\ 3.0 & 19784.8 \\ 4.0 & 19785.2 \\ \text { 5. } & 19782.1 \\ 6.0 & 19780.5 \\ 7.0 & 19781.1 \\ \text { 8. } 0 & 19779.4 \\ \text { 7.0 } & 19782.2 \\ 10.0 & 19781.1 \\ 11.0 & 19778.5 \\ 12.0 & 19779.4 \\ 13.0 & 19779.2 \\ 14.0 & 19779.1 \\ 15.0 & 19780.2 \\ 16.0 & 19781.0 \\ 170 & 19780.7\end{array}$

NMR SCAN 4-SEP-1986 09:49:34.64

$\begin{array}{ccc}Z & \text { CURRENT } & \text { GAUSS/AMP } \\ 696.0 & 1922.6 & 10.2925 \\ 695.0 & 1922.6 & 10.2912 \\ 694.0 & 1922.6 & 10.2907 \\ 693.0 & 1922.7 & 10.2903 \\ 692.0 & 1922.6 & 10.2893 \\ 691.0 & 1922.6 & 10.2884 \\ 690.0 & 1922.6 & 10.2887 \\ 689.0 & 1922.7 & 10.2873 \\ 688.0 & 1922.6 & 10.2893 \\ 687.0 & 1922.6 & 10.2887 \\ 686.0 & 1922.6 & 10.2874 \\ 685.0 & 1922.6 & 10.2879 \\ 684.0 & 1922.6 & 10.2877 \\ 683.0 & 1922.7 & 10.2872 \\ 682.0 & 1922.8 & 10.2872 \\ 681.0 & 1922.7 & 10.2882 \\ 680.0 & 1922.8 & 10.2875 \\ 679.0 & 1922.9 & 10.2867 \\ & -92.9 & 10.2870\end{array}$

TIME

4-SEP-1986 09:39:57.89

4-SEP-1986 09:40:19.63

4-SEP-1986 09: 40:37.18

4-SEP-1986 09: 40:54. 88

4-SEP-1986 09:41:07.06

4-SEP-1986 09: $41: 20.76$

4-SEP-1986 09: $41: 33.96$

4-SEP-1986 09:41:45.37

4-SEP-1986 09: 42:03. 88

4-SEP-1986 09:42:19.36

4-SEP-1986 09: 42: 29. 86

4-SEP-1986 09: 42: 43.47

4-SEP-1986 09: 43:00. 46

4-SEP-1986 09: 43: 53. 21

4-SEP-1986 09: $44: 21.89$

4-SEP-1986 09: 44:47.49

4-SEP-1986 09: 45:01.85

4-SEP-1986 09:45:17.92

4-SEP-1986 09: 45:33. 91

4-SEP-1986 09: 45: 46.33

Figure 7 Beginning of typical NMR data file. 